Trends in Immunology Ms No. TREIMM-D-16-00019 (Revision)

\title{
Feedback amplification of neutrophil function
}

Tamás Németh and Attila Mócsai

Department of Physiology, Semmelweis University School of Medicine, 1094 Budapest, Hungary; MTA-SE "Lendület" Inflammation Physiology Research Group of the Hungarian Academy of Sciences and Semmelweis University, 1094 Budapest, Hungary

Running title: Neutrophil amplification loops

Correspondence should be addressed to:

nemeth.tamas@med.semmelweis-univ.hu (T. N.) or mocsai.attila@med.semmelweis-univ.hu (A. M.)

Corresponding author for editorial purposes:

Attila Mócsai, MD, PhD

Department of Physiology

Semmelweis University School of Medicine

Tuzolto utca 37-47, 1094 Budapest, Hungary

Tel: +36-1-459-1500 x60-409

Fax: $+36-1-266-7480$

E-mail: mocsai.attila@med.semmelweis-univ.hu 


\section{ABSTRACT}

As the first line of innate immune defense, neutrophils need to mount a rapid and robust antimicrobial response. Recent studies implicate various positive feedback amplification processes in achieving that goal. Feedback amplification ensures effective migration of neutrophils in shallow chemotactic gradients, multiple waves of neutrophil recruitment to the site of inflammation, as well as the augmentation of various effector functions of the cells. Here, we review such positive feedback loops including intracellular and autocrine processes, paracrine effects mediated by lipid $\left(\mathrm{LTB}_{4}\right)$, chemokine and cytokine mediators, and bidirectional interactions with the complement system and other immune and non-immune cells. Those amplification mechanisms are not only involved in antimicrobial immunity but also contribute to neutrophil-mediated tissue damage under pathological conditions. 


\section{OVERVIEW OF FEEDBACK AMPLIFICATION OF NEUTROPHIL FUNCTION}

Homeostatic mechanisms mostly rely on negative feedback mechanisms. However, rapid and robust responses to external stimuli often require positive feedback mechanisms. Typical examples for positive feedback include action potential, ovulation or blood clotting, all of which result in dramatic changes in biological processes.

Neutrophils form the first line of innate immune defense against bacterial and fungal pathogens [1]. They are equipped with a multitude of cell surface receptors for the recognition of microbial pathogens and the inflammatory environment [2], and migrate to the site of inflammation through a multistep recruitment process triggered by chemotactic agents including chemokines, lipid chemoattractants (primarily $\mathrm{LTB}_{4}$ ), formyl-peptides and complement fragments [3-5]. At the site of infection/inflammation, neutrophils use an armamentarium of effector functions, including phagocytosis, respiratory burst, degranulation and neutrophil extracellular trap (NET) formation to eliminate the invading microorganisms [6,7]. In addition, neutrophils also participate in the organization of the overall immune response [6-9]. Despite being short-lived, terminally differentiated cells with limited transcriptional activity, they can undergo stimulus-induced gene expression changes leading to chemokine and cytokine release into the extracellular space $[6,10,11]$. Given their robust effector functions, neutrophils also play a major role in tissue damage during infectious or non-infectious inflammatory diseases [6,12].

A number of recent reports indicate important roles for self-perpetuating positive feedback amplifications loops in promoting neutrophil recruitment and activation. Such feedback loops act at various levels, including amplification within single cells, paracrine interaction between different neutrophils, as well as more complex feedback loops involving other biochemical and cellular processes (Figure 1). Those amplification loops have likely evolved to ensure swift and robust responses against invading microorganisms but they also contribute to neutrophil-mediated tissue damage during infectious and sterile inflammatory diseases. Here we review the 
various mechanisms of feedback amplification of neutrophil function and their relevance to neutrophil-mediated in vivo biological processes. Understanding feedback amplification of neutrophil function may facilitate the development of novel therapeutic strategies in diseases characterized by excessive neutrophil activation.

\section{FEEDBACK AMPLIFICATION AT THE SINGLE CELL LEVEL}

Recent reports indicate that the function of single neutrophils is amplified by several intracellular or autocrine feedback amplification pathways.

\section{Intracellular feedback loops promoting neutrophil polarization}

One of the most prominent biochemical features of neutrophil polarization is the accumulation of $\mathrm{Ptd} \operatorname{lns}(3,4,5) \mathrm{P}_{3}\left(\mathrm{PIP}_{3}\right)$ at the leading edge. The cellular $\mathrm{PIP}_{3}$ gradient is substantially steeper than that of the extracellular chemoattractant gradient and exogenous $\mathrm{PIP}_{3}$ leads to the accumulation of endogenous $\mathrm{PIP}_{3}$ at the leading edge [13], suggesting the involvement of molecular amplification processes. Chemoattractant receptors trigger initial $\mathrm{PIP}_{3}$ production at the leading edge through PI3-kinases activated by G-protein $\beta y$ subunits (Figure 2). This initial $\mathrm{PIP}_{3}$ production leads to activation of Rac small GTPases, which then activate the WAVE2 complex scaffolded by the Hem-1 protein, eventually leading to actin polymerization and activation of further endogenous PI3-kinases at the leading edge [13-15]. In addition, Rac GTPases also activate the NADPH oxidase and reactive oxygen species (ROS) production at the leading edge. This results in local inhibition of PTEN, a lipid phosphatase responsible for the degradation of $\mathrm{PIP}_{3}$, leading to further accumulation of $\mathrm{PIP}_{3}$ at the leading edge [16]. Those mechanisms (Figure 2) contribute to the translation of a shallow extracellular gradient to robust neutrophil polarization.

While the leading edge is characterized by Rac activation and actin polymerization-based protrusions, the trailing edge (uropod) is dominated by Rho 
activation and myosin-based contraction through the Rho-activated kinase ROCK which phosphorylates and activates the myosin light chain MLC [5]. This differential signaling defines the biochemical basis of "frontness" and "backness" in polarized neutrophils (Figure 2). Interestingly, the two domains are mutually exclusive due to mutual inhibition of each other at the biochemical level [17]. Constitutive activation of the uropod components Rho, ROCK or MLC inhibited establishment of leading-edge features such as $\mathrm{PIP}_{3}$ accumulation, Rac activation or actin polymerization, while inhibition of the Rho/ROCK/myosin pathway had the opposite effect [17]. Furthermore, inhibition of "frontness" features such as actin polymerization or Gi activation [18], or by knockdown of the leading edge organizer Hem-1 [15], inhibited "backness" feature such as uropod-specific localization of Rho and phosphorylated MLC. The reciprocal inhibition between "frontness" and "backness" features (i. e. the inhibition of the inhibitors) provides an additional level of positive feedback amplification of neutrophil polarization and contributes to the rapid migration of neutrophils. It should be noted that PI3-kinase mediated cell polarization and migration is not unique to neutrophils in the immune system, as it also exists in T cells and macrophages $[19,20]$.

\section{Feedback amplification through autocrine loops}

Neutrophil migration is also promoted through autocrine feedback amplification loops (Fig 2). Neutrophils release ATP into the extracellular space from their leading edge [21] which then becomes hydrolyzed by ecto-ATPases to adenosine. ATP activates $\mathrm{P} 2 \mathrm{Y} 2$ receptors at the leading edge to promote neutrophil polarization, whereas adenosine activates $\mathrm{A} 3$ receptors to promote migration of the cells along the chemotactic gradient [21]. Accordingly, P2Y2 and A3 receptors are required for effective directional migration of neutrophils both in vitro and in vivo [21]. ATP release also amplifies non-chemotactic functions of neutrophils through ligation of P2Y2 receptors on the same cells [22]. Taken together, autocrine activation of purinergic 
receptors contributes to robust neutrophil migration and activation responses, reminiscent of the role of IL-2 in T cell activation.

Integrin-mediated adhesion as a positive feedback loop

The majority of neutrophil functions requires adhesion to endothelial cells or extracellular matrix proteins through $\beta_{2}$ integrins. Integrins are bidirectional signaling molecules which relay extracellular signals to the inside of the cell ("outside-in" signaling) but their ligand binding capacity (affinity or avidity) is also regulated by intracellular signals ("inside-out" signaling). Initial ligation of integrins triggers outside-in signals and functional responses through an immunoreceptor-like signaling pathway [23-31]. While integrin signaling routes mediate adhesion and/or spreading of neutrophils [23-27], they also trigger inside-out signaling responses under certain conditions [32-36]. The sequential activation of outside-in and inside-out integrin signaling results in feedback amplification of integrin-mediated neutrophil function.

\section{PARACRINE MECHANISMS OF FEEDBACK AMPLIFICATION}

Neutrophils have also been shown to release extracellular mediators that promote the function of adjacent neutrophils in a paracrine manner. Those mediators may either be bioactive lipids or proteinaceous (chemokine, cytokine) substances.

\section{Amplification through neutrophil-derived $L T B_{4}$}

The effect of the neutrophil-derived lipid product $\mathrm{LTB}_{4}$ on other neutrophils provides one of the best examples of feedback amplification of neutrophil function. Neutrophils are the most prominent source and one of the most prominent targets of $\mathrm{LTB}_{4}$ [37]. $\mathrm{LTB}_{4}$ accumulates at the site of inflammation in the autoantibody-induced $\mathrm{K} / \mathrm{B} \times \mathrm{N}$ serum-transfer arthritis model $[38,39]$. Mice lacking critical $\mathrm{LTB}_{4}$ synthesis enzymes (5-lipoxygenase or $\mathrm{LTA}_{4}$ hydrolase) or the high-affinity $\mathrm{LTB}_{4}$ receptor BLT1 
are protected from neutrophil accumulation and development of autoantibody-induced arthritis $[38,40,41]$. Importantly, adoptive transfer of wild type neutrophils restored arthritis development in 5-lipoxygenase deficient $\left(A l o x 5^{--}\right)$and BLT1-deficient $\left(L t b 4 r 1^{-}\right.$ ${ }^{-}$) recipients $[38,40,41]$, indicating that neutrophils are both a major cellular source and the principal target of $\mathrm{LTB}_{4}$ in this model (Figure 3). This was in line with the immune complex-induced in vitro release of $\mathrm{LTB}_{4}$ from neutrophils but not macrophages $[11,39,41,42]$. Further signaling studies (Figure 3B) have also revealed that the $\mathrm{LTB}_{4}{ }^{-}$ mediated feedback amplification of neutrophil recruitment was mediated by Src-family kinases, Syk, PLCY2 and, likely, Vav-family members, but not by the CARD9 adapter protein $[11,27,39,43,44]$.

In a laser-induced sterile dermal inflammation model, the initial accumulation of resident neutrophils was followed by a second, larger wave of neutrophil recruitment and clustering [45]. This second wave, termed neutrophil "swarming" because of its resemblance of the coordinated movement of insect swarms $[45,46]$, was diminished in $L t b 4 r 1^{-1-}$ and $A l o x 5^{-/-}$mice [45]. Interestingly, when wild type and $L t b 4 r 1^{-/-}$neutrophils

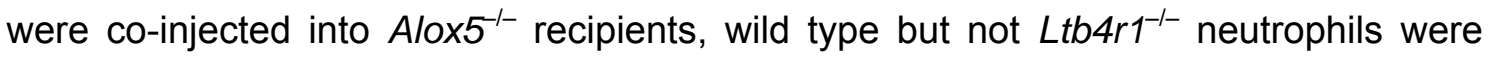
able to accumulate at the site of injury, suggesting that neutrophils release $\mathrm{LTB}_{4}$ which triggers recruitment of further neutrophils through BLT1 ligation [45]. Those results provided further evidence for $\mathrm{LTB}_{4}$-mediated amplification of neutrophil recruitment.

$\mathrm{LTB}_{4}$-mediated amplification of neutrophil recruitment has also been reported in other in vivo inflammation models. Neutrophil-derived $\mathrm{LTB}_{4}$ accumulated at the inflammatory site in a novel model of atopic dermatitis [47]. Importantly, $L t b 4 r 1^{-1-}$ neutrophils were unable to accumulate at the inflammation site [47], indicating $\mathrm{LTB}_{4}$ mediated feedback amplification also in this model (Figure 3). BLT1-dependent neutrophil clustering was also observed in a Pseudomonas aeruginosa infection model [45], and BLT1 was also required for neutrophil recruitment and antibody-mediated tumor immunotherapy in experimental lymphoma [48]. 
Analysis of formyl-peptide-induced in vitro migration of neutrophils provided further evidence for an $\mathrm{LTB}_{4}$-mediated amplification loop [49]. Formyl peptides triggered chemotactic migration of wild type neutrophils but not those lacking the predominant formyl-peptide receptor FPR1. Inhibition of 5-lipoxygenase or genetic deletion of 5-lipoxygenase or BLT1 attenuated formyl-peptide-induced neutrophil migration [49]. Surprisingly, even $\mathrm{Fpr}^{-1-}$ neutrophils were able to migrate towards formyl peptides when they were mixed with wild type neutrophils. However, no migration of $\mathrm{FprI}^{-1-}$ neutrophils could be observed in the presence of 5 -lipoxygenase

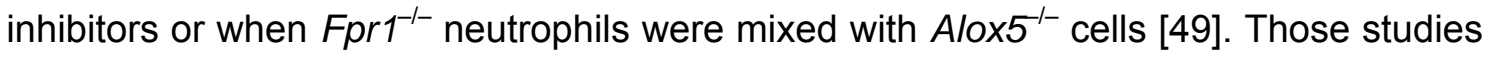
suggest that formyl peptides trigger a secondary gradient of $\mathrm{LTB}_{4}$ which is able to direct the migration of even those cells that cannot sense the primary gradient. Transepithelial migration of neutrophils towards Pseudomonas aeruginosa also required neutrophil-derived $\mathrm{LTB}_{4}$ acting through the BLT1 receptor [50].

Neutrophil-derived $\mathrm{LTB}_{4}$ also amplifies non-migratory functions of neutrophils. Immune complex stimulation triggered robust release of $\operatorname{LTB}_{4}$ from neutrophils $[11,39]$ and the respiratory burst under such conditions was reduced by inhibitors of 5lipoxygenase or BLT1 [42]. On the other hand, inhibition of 5-lipoxygenase or BLT1 did not affect adhesion or spreading of formyl-peptide-stimulated neutrophils, indicating the specificity of the $\mathrm{LTB}_{4}$-mediated amplification loop [49].

A very recent report provided interesting mechanistic insight into $L T B_{4}$-mediated feedback amplification [51]. Both $\mathrm{LTB}_{4}$ and its synthesizing enzymes 5-lipoxygenase, LTA $_{4}$-hydrolase and FLAP were localized to multivesicular bodies and released in form of extracellular vesicles upon fMLP-induced neutrophil activation [51]. Exosomederived $\mathrm{LTB}_{4}$ acted both on the originating as well as on neighboring cells to promote neutrophil migration towards an fMLP source [51]. Those results extend prior reports [49] and suggest that $\mathrm{LTB}_{4}$ exerts its effect at least in part through neutrophil-derived extracellular vesicles (Figure 3). 
In conclusion, the lipid mediator $\mathrm{LTB}_{4}$ directs neutrophils towards the site of inflammation and augments their cellular responses by several BLT1-dependent paracrine mechanisms including the potentiation of fMLP receptor signaling, contribution to immune complex-activation and acting of neutrophil-derived microvesicles on adjacent neutrophils.

\section{Amplification by neutrophil-derived chemokines}

Neutrophil-derived chemokines are also involved in paracrine amplification of neutrophil function (Figure 3 ). In the $\mathrm{K} / \mathrm{B} \times \mathrm{N}$ serum-transfer arthritis model, adoptive transfer of wild type neutrophils into $L t b 4 r 1^{-/-}$mice surprisingly also restored the recruitment of $L t b 4 r 1^{-/-}$recipient neutrophils [40], suggesting that activated neutrophils are able to recruit other neutrophils through factors other than $\mathrm{LTB}_{4}$. In addition, germline or neutrophil-specific deletion of the CARD9 gene expression regulator reduced autoantibody-induced arthritis and dermatitis, along with blocking chemokine and cytokine but not $\mathrm{LTB}_{4}$ release from neutrophils [11]. Indeed, immune complexinduced neutrophil activation triggered the release of a number of chemokines that are able to attract neutrophils, the most important of which is CXCL2 (MIP-2), but CXCL1 (KC), and CCL3 (MIP-1 $\alpha$ ) also have effects on neutrophils $[11,39,52]$. While $\mathrm{LTB}_{4}$ was involved in the early phase of autoantibody-induced arthritis, neutrophil-derived chemokines promoted the recruitment of additional neutrophils during later stages of arthritis development as another feedback amplification process [52]. Additional signaling studies (Figure 3B) revealed that the chemokine-mediated feedback amplification of neutrophil recruitment was mediated by the CARD9 gene expression regulator acting downstream of Src-family kinases, Syk, PLCY2 and, likely, Vav-family members $[11,27,39,43,44]$. 


\section{Other paracrine amplification mechanisms}

Neutrophils release a number of additional molecules that are able to activate other neutrophils in a paracrine manner (Figure 3A). Myeloperoxidase, released from the primary (azurophilic) granules of neutrophils, binds to neutrophil Mac-1 and enhances respiratory burst and granule release [53]. Myeloperoxidase also attracts neutrophils by promoting their adhesion to endothelial cells through electrostatic interactions [54]. Importantly, neither of those responses required peroxidase activity [53,54], indicating a non-enzymatic action of myeloperoxidase. Neutrophil activation also triggers release of reactive oxygen species which are thought to amplify various neutrophil functions through the activation of tyrosine phosphorylation pathways and, possibly, other mechanisms [55,56]. Neutrophils also release adenosine which may enhance (but may also inhibit) neutrophil functions through different adenosine receptors [57]. In addition, neutrophil-derived TNF- $\alpha$ has been shown to contribute to the amplification of IL- 6 production by human neutrophils stimulated by a TLR8 ligand $[58]$.

The MRP8/14 (S100A8/9) proteins are the most abundant cytosolic proteins in neutrophils and are released into the extracellular space upon neutrophil activation [59]. Importantly, MRP8/14 has been shown to promote neutrophil adhesion and, possibly, other neutrophil functions $[60,61]$. A recent report also revealed that MRP8/14 is released upon neutrophil rolling on E-selectin-coated endothelial surfaces and then promotes rapid activation of $\beta_{2}$ integrin-mediated neutrophil adhesion by binding to the TLR4 receptor [62], contributing to the amplification of neutrophil recruitment.

Taken together, several paracrine mechanisms contribute to positive feedback loops of neutrophils, including the lipid mediator $\mathrm{LTB}_{4}$, chemokines, the granule component myeloperoxidase or MRP8. Those mediators act on different receptors including the $\mathrm{LTB}_{4}$ receptor BLT1, G protein-coupled chemokine receptors, as well as integrins and Toll-like receptors. 


\section{COMPLEX FEEDBACK AMPLIFICATION LOOPS}

Neutrophils are also involved in more complex feedback amplification loops involving complex biochemical pathways or interaction with other cell types.

\section{Amplification of neutrophil function through complement activation}

The complement system interacts with neutrophils in a bidirectional manner. While neutrophils are capable of promoting complement activation, end products of complement activation such as $\mathrm{C} 5 \mathrm{a}$, a major neutrophil chemoattractant, and $\mathrm{C} 3 \mathrm{~b}$, a ligand of the $\beta_{2}$-integrin Mac-1, also strongly stimulate neutrophils (Figure 4A).

The alternative pathway of complement activation mediates autoantibodyinduced arthritis through the action of complement-derived C5a on C5a-receptors on neutrophils $[41,63,64]$. C5a is able to promote $\mathrm{LTB}_{4}$ production by neutrophils $[41]$ and mixed bone marrow chimeric and adoptive neutrophil transfer experiments revealed that C5a-receptors and 5-lipoxygenase had to be present on the same individual neutrophils [41]. Those results suggest that neutrophils act downstream of complement activation through $\mathrm{C} 5 \mathrm{a}$-mediated $\mathrm{LTB}_{4}$ production. However, several lines of evidence suggest that neutrophils are also required for complement activation in this model. In the affected joints, neutrophils but not $\mathrm{C} 3$ or $\mathrm{C} 5 \mathrm{a}-$-receptors were required for the initial vascular permeability increase [65] and neutrophils but not C5 were required for the initial accumulation of pathogenic anti-GPI antibodies [66]. Both those initial responses also required the Fc-receptor $\mathrm{y}$-chain which is thought to be primarily involved in neutrophil activation in this model [64-66]. Autoantibody-induced accumulation of C5/C5a was also abrogated in mice lacking the myeloid-specific Src-family kinases Hck, Fgr and Lyn which likely mediate neutrophil activation in this model [39]. Those results together suggest complement-mediated amplification of neutrophil function in autoantibody-induced arthritis and, possibly, in other autoantibody-mediated (e. g. glomerulonephritis, vasculitis, dermatitis) diseases (Figure 4A). 
In vitro studies also confirmed the complement-mediated feedback amplification of neutrophil function. Supernatants of neutrophils activated by anti-neutrophil cytoplasmic antibodies triggered complement activation and C5a generation $[67,68]$. Such conditioned supernatants were then able to prime neutrophils for respiratory burst in a C5a-receptor-dependent manner, indicating existence of a feedback amplification loop [68]. Neutrophil activation by various stimuli also triggered the alternative pathway of complement activation which in turn resulted in neutrophil activation primarily through C5a generation [69].

The mechanism of neutrophil-mediated complement activation is still incompletely understood. While prior studies suggested a role for reactive oxygen species and granule enzymes such as myeloperoxidase and neutrophil-derived proteases [67], those assumptions could not be confirmed by a more recent study [69]. On the other hand, activated neutrophils have been shown to release properdin, a positive regulator of the alternative complement pathway $[69,70]$. Together with the role of properdin expressed in a myeloid-lineage cell in the development of the $K / B \times N$ serum-transfer arthritis [71], those results suggest amplification of neutrophil function through complement activation triggered by neutrophil-derived properdin (Figure 4A).

Taken together, the activation of the complement system is a critical feedback amplification mechanism of neutrophil function since the cascade can be initiated by neutrophils and its products ( $\mathrm{C} 5 \mathrm{a}$ and $\mathrm{C} 3 \mathrm{~b})$ also act on neutrophils themselves, either as chemoattractants or activators of the cells.

\section{Amplification by granule proteases}

Neutrophils contain several granule proteins, including proteolytic enzymes such as the matrix metalloprotease gelatinase B (MMP9) or neutrophil elastase that are released upon cellular activation. In line with the role of matrix metalloproteases in processing various chemokines and cytokines [72], gelatinase B is able to cleave CXCL8/IL-8, strongly increasing its potency to further activate neutrophils [73]. 
Gelatinase B also triggers the generation of Pro-Gly-Pro (PGP) peptide fragments from extracellular matrix proteins that attract and activate neutrophils through CXCR $1 / 2$ during airway inflammation (Figure 4B) $[74,75]$. Neutrophil proteases have also been found to self-perpetuate the pathogenesis of experimental bullous pemphigoid: upon neutrophil activation, gelatinase $B$ is released and activates neutrophil elastase by cleaving its natural inhibitor $\alpha_{1}$ proteinase inhibitor; elastase then digests BP180, generating fragments with direct chemotactic activity on other neutrophils [76-78].

Taken together, granule proteases are not only important components of antimicrobial activity, but they also actively participate in augmenting neutrophil functions by increasing the potency of neutrophil-active chemokines or generating chemoattractants from extracellular proteins.

\section{Amplification of neutrophil function through other immune cells}

Neutrophils participate in bidirectional interactions with other immune cells [79]. Neutrophils can recruit and activate inflammatory monocytes/macrophages and vice versa. Both neutrophils and monocytes/macrophages can produce several chemokines like CXCL1, CXCL2, CXCL8, CCL2, CCL3 and CCL4 chemokines besides expressing their receptors [80]. Furthermore, neutrophil granule components like cathepsin G or azurocidin also attract monocytes [81]. Azurocidin exerts its recruitment effect directly through $\beta_{2}$ integrins or indirectly through endothelial cells and activates macrophages to release neutrophil degranulating cytokines, completing a feedback amplification loop [82] (Figure 4C). Activated macrophages can also release matrix metalloproteinases [83] such as MMP8 and MMP9, which promote neutrophil chemotaxis by proteolytic activation of CXCL5 and CXCL8 [73,84]. Moreover, macrophages, in concert with TNFa, can trigger CCL2 (MCP-1) release from neutrophils and promote their own recruitment [85]. Further details of the interaction of neutrophils and monocytes/macrophages can be found in refs. $[80,86]$. 
Th17 cells are crucial in antifungal host defense and in various autoimmune diseases. A recent study showed that neutrophils stimulated by IFNY and LPS release Th17 cell-attracting CCL2 and CCL20 chemokines, while activated Th17 cells produce the neutrophil chemoattractant CXCL8 [87] (Figure 4C). In line with this bidirectional recruitment effect, both neutrophils and Th17 cells were found in the synovial fluid of patients with rheumatoid arthritis and in gut samples from people with Crohn's disease [87]. Moreover, Th17 cells promote granulopoiesis via the release of G-CSF from tissue fibroblasts and can delay neutrophil apoptosis through the production of GMCSF, TNFa and IFNY $[87,88]$.

The pathogenesis of atherosclerosis serves as an example for a complex amplification loop between neutrophils, macrophages and Th17 cells. Cholesterol crystals, which are typical constituents of advanced atherosclerotic plaques, can trigger macrophage IL-1 $\beta$ and IL-6 production through NET formation, leading to the release of IL-17 from Th17 cells. IL-17 then triggers the release of neutrophil chemoattractants (e. g. CXCL2), resulting in the amplification of neutrophil recruitment and atherosclerosis progression in mice [89]. However, some authors argue against the importance of NET formation in the development of atherosclerosis, pointing to other potential neutrophilmediated mechanisms [90].

Certain bacterial antigens can trigger neutrophils to produce mature IL-18 that promotes the release of IFNy from NK cells in the presence of dendritic cell-derived IL12 [91]. IL-18-activated NK cells can prolong neutrophil survival through the production of GM-CSF and IFNy [92]. For further cooperative amplification loops between neutrophils and T cells or NK cells, we refer to previous review articles $[79,93]$.

The role of platelets and stromal cells in the amplification of neutrophil function

Endothelium-attached activated platelets initiate a neutrophil-platelet interaction mainly through PSGL-1-P-selectin and LFA-1-ICAM-2 interactions promoting neutrophil extravasation [94]. Activated platelets also release CXCL4 and CCL5 from 
their a-granules that promote neutrophil accumulation in septic lungs in the coecal ligation and puncture model through the release of CXCL2 from alveolar macrophages $[95,96]$. On the other hand, neutrophil-derived cathepsin $G$ can trigger platelet activation through the protease-activated receptor 4 (PAR4) and mediate intracellular calcium signaling [97]. Moreover, cathepsin G can cause platelet aggregation [98]. In the presence of LPS, platelets provoke NET formation, which activates the coagulation cascade with an increase in the local platelet-activating thrombin concentration [99]. Moreover, NETs can directly activate platelets [99] (Figure 4D). For further information, we refer to previous review articles [100,101].

In experimental arthritis, immune complex-activated neutrophils undergo gene expression changes and produce interleukin- $1 \beta$ that provokes resident synovial tissue cells like fibroblast-like synoviocytes to produce neutrophil attracting chemokines $[52,64]$. Fibroblast-like synoviocytes mainly attract neutrophils by producing CXCL5, CXCL1 and CXCL2 [52] (Figure 4E).

There are several ways for endothelial cells to activate neutrophils and vice versa. Activated endothelial cells promote mobilization of neutrophils from the bone marrow through G-CSF, recruit them by chemokines (e.g. by CXCL1 and CXCL2), and participate in neutrophil extravasation; inflammatory neutrophils in turn produce cytokines (e.g. IL-1 $\beta$, TNF $\alpha$ ) that activate the vascular endothelium [52,102] (Figure $4 \mathrm{E})$.

Danger-associated molecular patterns (DAMPs) such as ATP or uric acid can be released from dying stromal cells and can trigger neutrophil migration/activation [103]. As neutrophil influx often causes tissue injury, it is probable that necrosis-inducing neutrophils support their own recruitment and/or activation through these molecules.

In conclusion, platelets and different types of stromal cells act in concert to trigger amplification of neutrophil activation and enhance their effector functions. 


\section{Additional complex amplification of neutrophil function}

The pathogenesis of systemic lupus erythematosus is a typical example for a feedback amplification involving neutrophils and dendritic cells. DNA-anti-DNA immune complexes trigger the release of NETs, increasing the amount of extracellular DNA molecules $[104,105]$. This process is amplified by immune complexes containing neutrophil-derived DNA decorated with the antimicrobial peptide LL37, which activates plasmacytoid dendritic cells to release interferon- $\alpha$ (IFNa); IFNa then facilitates NET formation, amplifying the initial disease process (Figure 4F) [104,105].

In small vessel vasculitis, anti-neutrophil cytoplasmic antibodies (ANCA) can trigger the release of the B cell survival factor B Lymphocyte Stimulator (BLyS) from neutrophils, which, besides serving as a survival signal, helps the differentiation of B cells into ANCA-producing plasma cells [106]. These pathological anti-neutrophil cytoplasmic antibodies (ANCA) targeting myeloperoxidase or proteinase 3 can then trigger NET formation, which results in the release of further myeloperoxidase and proteinase 3 autoantigens (Figure 4F) [107].

Similar to ANCA, anti-citrullinated protein antibodies (ACPA) found in the most progressive forms of rheumatoid arthritis has been shown to provoke neutrophil NET formation, leading to release of citrullinated autoantigens and generation of further ACPA-containing immune complexes (Figure 4F) [108]. Furthermore, these NETs could induce CXCL8 release from synovial fibroblasts [108].

In summary, positive neutrophil amplification loops also seem to be crucial components of human autoimmune disorders.

\section{CONCLUSION}

The feedback amplification mechanisms discussed above are well suited to promote a rapid and robust neutrophil response during the early phases of microbial invasion. Feedback amplification may enhance neutrophil function at several different 
levels. It may promote sensing and responding to minute changes and small gradients in the extracellular environment and it may augment the response of neutrophils by increasing the speed, amplitude and range of the response. At the cellular level, positive feedback triggers the self-organization of cellular responses such as polarization or lamellipodium formation.

A typical feature of feedback amplification is that the entire process is abrogated irrespective of where the loop is broken, providing an opportunity for therapeutic intervention (see Outstanding Questions). However, this also often conceals exact molecular mechanisms and hierarchies between the components involved. Given the strong feedback amplification of neutrophil function, one would expect that terminating the inflammation process would also require active mechanisms. Indeed, the last several years have revealed that termination of inflammation is governed by an active process called resolution of inflammation, coordinated by specialized pro-resolving lipid mediators [109].

Besides understanding the overall mechanisms of feedback amplification of neutrophil function, several reports have also contributed to understanding the signaling details involved in an in vivo inflammatory environment. We and others have shown that a tyrosine kinase signaling pathway involving Src-family kinases, Syk, PLCY2 and CARD9 plays a critical role in neutrophil-mediated inflammatory reactions and proposed that they are primarily involved in triggering processes that amplify neutrophil function through the generation of extracellular inflammatory mediators $[11,27,39,43,44,110]$. Components of that pathway may become therapeutic targets of inflammatory diseases involving a robust neutrophil response [12].

Though feedback amplification is likely critical for the swift and robust responses of neutrophils to invading microorganisms, it also contributes to neutrophil-mediated tissue damage. Understanding feedback amplification processes may therefore provide better understanding and possibly novel therapeutic targets of diseases characterized by a significant neutrophil-mediated inflammatory component. 


\section{ACKNOWLEDGMENT}

We apologize to colleagues whose work could not be discussed due to space limitation. Work in the authors' laboratory has been funded by the "Lendület" program of the Hungarian Academy of Sciences (LP2013-66 to A. M.). A. M. was a recipient of a

Wellcome Trust International Senior Research Fellowship (Grant No. 087782). T. N. was a recipient of a János Bolyai Research Scholarship from the Hungarian Academy of Sciences.

\section{CONFLICT OF INTEREST DISCLOSURES}

The authors have no conflicting financial interests. 


\section{REFERENCES}

1. Nathan, C. (2006) Neutrophils and immunity: challenges and opportunities. Nat Rev Immunol 6, 173-182.

2. Futosi, K., et al. (2013) Neutrophil cell surface receptors and their intracellular signal transduction pathways. Int Immunopharmacol 17, 638-650.

3. Kolaczkowska, E., et al. (2013) Neutrophil recruitment and function in health and inflammation. Nat Rev Immunol 13, 159-175.

4. Nourshargh, S., et al. (2014) Leukocyte migration into inflamed tissues. Immunity 41, 694707.

5. Mócsai, A., et al. (2015) Intracellular signalling during neutrophil recruitment. Cardiovasc Res 107, 373-385.

6. Mócsai, A. (2013) Diverse novel functions of neutrophils in immunity, inflammation, and beyond. J Exp Med 210, 1283-1299.

7. Mayadas, T.N., et al. (2014) The multifaceted functions of neutrophils. Annu Rev Pathol 9, 181-218.

8. Mantovani, A., et al. (2011) Neutrophils in the activation and regulation of innate and adaptive immunity. Nat Rev Immunol 11, 519-531.

9. Weber, F.C., et al. (2015) Neutrophils are required for both the sensitization and elicitation phase of contact hypersensitivity. J Exp Med 212, 15-22.

10. Tecchio, C., et al. (2014) Neutrophil-derived cytokines: facts beyond expression. Front Immunol 5, 508.

11. Németh, T., et al. (2016) Neutrophil-specific deletion of the CARD9 gene expression regulator suppresses autoantibody-induced inflammation in vivo. Nat Commun 7, 11004.

12. Németh, T., et al. (2012) The role of neutrophils in autoimmune diseases. Immunol Lett 143, 9-19.

13. Weiner, O.D., et al. (2002) A Ptdlns $\mathrm{P}_{3^{-}}$and Rho GTPase-mediated positive feedback loop regulates neutrophil polarity. Nat Cell Biol 4, 509-513.

14. Wang, F., et al. (2002) Lipid products of PI3Ks maintain persistent cell polarity and directed motility in neutrophils. Nat Cell Biol 4, 513-518.

15. Weiner, O.D., et al. (2006) Hem-1 complexes are essential for Rac activation, actin polymerization, and myosin regulation during neutrophil chemotaxis. PLOS Biol 4, e38.

16. Kuiper, J.W., et al. (2011) Rac regulates $\mathrm{Ptdlns}_{3}$ signaling and the chemotactic compass through a redox-mediated feedback loop. Blood 118, 6164-6171.

17. Xu, J., et al. (2003) Divergent signals and cytoskeletal assemblies regulate self-organizing polarity in neutrophils. Cell 114, 201-214.

18. Wong, K., et al. (2006) Neutrophil polarization: spatiotemporal dynamics of RhoA activity support a self-organizing mechanism. Proc Natl Acad Sci USA 103, 3639-3644.

19. Turner, L., et al. (1995) RANTES-activated human T lymphocytes. A role for phosphoinositide 3-kinase. J Immunol 155, 2437-2444.

20. Hirsch, E., et al. (2000) Central role for $G$ protein-coupled phosphoinositide 3-kinase $\gamma$ in inflammation. Science 287, 1049-1053.

21. Chen, Y., et al. (2006) ATP release guides neutrophil chemotaxis via P2Y2 and A3 receptors. Science 314, 1792-1795.

22. Chen, Y., et al. (2010) Purinergic signaling: a fundamental mechanism in neutrophil activation. Sci Signal 3, ra45.

23. Lowell, C.A., et al. (1996) Deficiency of Src family kinases $p 59 / 61^{\text {hck }}$ and $p 58^{\text {c-fgr }}$ results in defective adhesion-dependent neutrophil functions. J Cell Biol 133, 895-910.

24. Mócsai, A., et al. (1999) Adhesion-dependent degranulation of neutrophils requires the Src family kinases Fgr and Hck. J Immunol 162, 1120-1126.

25. Mócsai, A., et al. (2002) Syk is required for integrin signaling in neutrophils. Immunity 16 , 547-558.

26. Newbrough, S.A., et al. (2003) SLP-76 regulates Fcy receptor and integrin signaling in neutrophils. Immunity 19, 761-769.

27. Jakus, Z., et al. (2009) Critical role of phospholipase Cy2 in integrin and Fc receptormediated neutrophil functions and the effector phase of autoimmune arthritis. $J$ Exp Med 206, 577-593.

28. Giagulli, C., et al. (2006) The Src family kinases Hck and Fgr are dispensable for inside-out, chemoattractant-induced signaling regulating $\beta_{2}$ integrin affinity and valency in neutrophils, 
but are required for $\beta_{2}$ integrin-mediated outside-in signaling involved in sustained adhesion. J Immunol 177, 604-611.

29. Graham, D.B., et al. (2007) Neutrophil-mediated oxidative burst and host defense are controlled by a Vav-PLCY2 signaling axis in mice. J Clin Invest 117, 3445-3452.

30. Jakus, Z., et al. (2007) Immunoreceptor-like signaling by $\beta_{2}$ and $\beta_{3}$ integrins. Trends Cell Biol 17, 493-501.

31. Mócsai, A., et al. (2010) The SYK tyrosine kinase: a crucial player in diverse biological functions. Nat Rev Immunol 10, 387-402.

32. Zarbock, A., et al. (2007) Spleen tyrosine kinase Syk is necessary for E-selectin-induced $\alpha_{\llcorner} \beta_{2}$ integrin-mediated rolling on intercellular adhesion molecule-1. Immunity 26, 773-783.

33. Mueller, H., et al. (2010) Tyrosine kinase Btk regulates E-selectin-mediated integrin activation and neutrophil recruitment by controlling phospholipase C (PLC) Y2 and PI3Ky pathways. Blood 115, 3118-3127.

34. Yago, T., et al. (2010) E-selectin engages PSGL-1 and CD44 through a common signaling pathway to induce integrin $\alpha_{\llcorner} \beta_{2}$-mediated slow leukocyte rolling. Blood 116, 485-494.

35. Block, H., et al. (2012) Crucial role of SLP-76 and ADAP for neutrophil recruitment in mouse kidney ischemia-reperfusion injury. J Exp Med 209, 407-421.

36. Volmering, S., et al. (2016) The Neutrophil Btk Signalosome Regulates Integrin Activation during Sterile Inflammation. Immunity.

37. Peters-Golden, M., et al. (2007) Leukotrienes. N Engl J Med 357, 1841-1854.

38. Chen, M., et al. (2006) Neutrophil-derived leukotriene $B_{4}$ is required for inflammatory arthritis. J Exp Med 203, 837-842.

39. Kovács, M., et al. (2014) The Src family kinases Hck, Fgr, and Lyn are critical for the generation of the in vivo inflammatory environment without a direct role in leukocyte recruitment. J Exp Med 211, 1993-2011.

40. Kim, N.D., et al. (2006) A unique requirement for the leukotriene $B_{4}$ receptor BLT1 for neutrophil recruitment in inflammatory arthritis. J Exp Med 203, 829-835.

41. Sadik, C.D., et al. (2012) Neutrophils orchestrate their own recruitment in murine arthritis through C5aR and FcyR signaling. Proc Natl Acad Sci USA 109, E3177-3185.

42. Kulkarni, S., et al. (2011) PI3K $\beta$ plays a critical role in neutrophil activation by immune complexes. Sci Signal 4, ra23.

43. Jakus, Z., et al. (2010) Genetic deficiency of Syk protects mice from autoantibody-induced arthritis. Arthritis Rheum 62, 1899-1910.

44. Cremasco, V., et al. (2008) Vav/Phospholipase Cy2-mediated control of a neutrophildependent murine model of rheumatoid arthritis. Arthritis Rheum 58, 2712-2722.

45. Lammermann, T., et al. (2013) Neutrophil swarms require $\mathrm{LTB}_{4}$ and integrins at sites of cell death in vivo. Nature 498, 371-375.

46. Chtanova, T., et al. (2008) Dynamics of neutrophil migration in lymph nodes during infection. Immunity 29, 487-496.

47. Oyoshi, M.K., et al. (2012) Leukotriene $B_{4}$-driven neutrophil recruitment to the skin is essential for allergic skin inflammation. Immunity 37, 747-758.

48. Allendorf, D.J., et al. (2005) C5a-mediated leukotriene B4-amplified neutrophil chemotaxis is essential in tumor immunotherapy facilitated by anti-tumor monoclonal antibody and $\beta$ glucan. J Immunol 174, 7050-7056.

49. Afonso, P.V., et al. (2012) $\mathrm{LTB}_{4}$ is a signal-relay molecule during neutrophil chemotaxis. Dev Cell 22, 1079-1091.

50. Pazos, M.A., et al. (2015) Distinct cellular sources of hepoxilin A3 and leukotriene B4 are used to coordinate bacterial-induced neutrophil transepithelial migration. J Immunol 194, 1304-1315.

51. Majumdar, R., et al. (2016) Exosomes Mediate $\mathrm{LTB}_{4}$ Release during Neutrophil Chemotaxis. PLoS Biol 14, e1002336.

52. Chou, R.C., et al. (2010) Lipid-cytokine-chemokine cascade drives neutrophil recruitment in a murine model of inflammatory arthritis. Immunity 33, 266-278.

53. Lau, D., et al. (2005) Myeloperoxidase mediates neutrophil activation by association with CD11b/CD18 integrins. Proc Natl Acad Sci USA 102, 431-436.

54. Klinke, A., et al. (2011) Myeloperoxidase attracts neutrophils by physical forces. Blood 117, 1350-1358.

55. Fialkow, L., et al. (2007) Reactive oxygen and nitrogen species as signaling molecules regulating neutrophil function. Free Radic Biol Med 42, 153-164. 
56. Pricop, L., et al. (2002) Redox regulation of Fcy receptor-mediated phagocytosis: implications for host defense and tissue injury. Antioxid Redox Signal 4, 85-95.

57. Barletta, K.E., et al. (2012) Regulation of neutrophil function by adenosine. Arterioscler Thromb Vasc Biol 32, 856-864.

58. Zimmermann, M., et al. (2015) Chromatin remodelling and autocrine TNFa are required for optimal interleukin-6 expression in activated human neutrophils. Nat Commun 6, 6061.

59. Ehrchen, J.M., et al. (2009) The endogenous Toll-like receptor 4 agonist S100A8/S100A9 (calprotectin) as innate amplifier of infection, autoimmunity, and cancer. J Leukoc Biol 86, 557-566.

60. Newton, R.A., et al. (1998) The human S100 protein MRP-14 is a novel activator of the $\beta_{2}$ integrin Mac-1 on neutrophils. J Immunol 160, 1427-1435.

61. Ryckman, C., et al. (2003) Proinflammatory activities of S100: proteins S100A8, S100A9, and S100A8/A9 induce neutrophil chemotaxis and adhesion. J Immunol 170, 3233-3242.

62. Pruenster, M., et al. (2015) Extracellular MRP8/14 is a regulator of $\beta_{2}$ integrin-dependent neutrophil slow rolling and adhesion. Nat Commun 6, 6915.

63. Ji, H., et al. (2002) Arthritis critically dependent on innate immune system players. Immunity $16,157-168$.

64. Monach, P.A., et al. (2010) Neutrophils in a mouse model of autoantibody-mediated arthritis: critical producers of $\mathrm{Fc}$ receptor $\mathrm{Y}$, the receptor for $\mathrm{C} 5 \mathrm{a}$, and lymphocyte function-associated antigen 1. Arthritis Rheum 62, 753-764.

65. Binstadt, B.A., et al. (2006) Particularities of the vasculature can promote the organ specificity of autoimmune attack. Nat Immunol 7, 284-292.

66. Wipke, B.T., et al. (2004) Staging the initiation of autoantibody-induced arthritis: a critical role for immune complexes. J Immunol 172, 7694-7702.

67. Xiao, H., et al. (2007) Alternative complement pathway in the pathogenesis of disease mediated by anti-neutrophil cytoplasmic autoantibodies. Am J Pathol 170, 52-64.

68. Schreiber, A., et al. (2009) C5a receptor mediates neutrophil activation and ANCA-induced glomerulonephritis. J Am Soc Nephrol 20, 289-298.

69. Camous, L., et al. (2011) Complement alternative pathway acts as a positive feedback amplification of neutrophil activation. Blood 117, 1340-1349.

70. Wirthmueller, U., et al. (1997) Properdin, a positive regulator of complement activation, is released from secondary granules of stimulated peripheral blood neutrophils. J Immunol $158,4444-4451$.

71. Kimura, Y., et al. (2010) Genetic and therapeutic targeting of properdin in mice prevents complement-mediated tissue injury. J Clin Invest 120, 3545-3554.

72. Van Lint, P., et al. (2007) Chemokine and cytokine processing by matrix metalloproteinases and its effect on leukocyte migration and inflammation. J Leukoc Biol 82, 1375-1381.

73. Van den Steen, P.E., et al. (2000) Neutrophil gelatinase B potentiates interleukin-8 tenfold by aminoterminal processing, whereas it degrades CTAP-III, PF-4, and GRO- $\alpha$ and leaves RANTES and MCP-2 intact. Blood 96, 2673-2681.

74. Weathington, N.M., et al. (2006) A novel peptide CXCR ligand derived from extracellular matrix degradation during airway inflammation. Nat Med 12, 317-323.

75. Gaggar, A., et al. (2008) A novel proteolytic cascade generates an extracellular matrixderived chemoattractant in chronic neutrophilic inflammation. J Immunol 180, 5662-5669.

76. Liu, Z., et al. (1998) Gelatinase B-deficient mice are resistant to experimental bullous pemphigoid. J Exp Med 188, 475-482.

77. Liu, Z., et al. (2000) The serpin a1-proteinase inhibitor is a critical substrate for gelatinase B/MMP-9 in vivo. Cell 102, 647-655.

78. Lin, L., et al. (2012) Neutrophil elastase cleaves the murine hemidesmosomal protein BP180/type XVII collagen and generates degradation products that modulate experimental bullous pemphigoid. Matrix Biol 31, 38-44.

79. Scapini, P., et al. (2014) Social networking of human neutrophils within the immune system. Blood 124, 710-719.

80. Silva, M.T. (2010) When two is better than one: macrophages and neutrophils work in concert in innate immunity as complementary and cooperative partners of a myeloid phagocyte system. J Leukoc Biol 87, 93-106.

81. Chertov, O., et al. (1997) Identification of human neutrophil-derived cathepsin $G$ and azurocidin/CAP37 as chemoattractants for mononuclear cells and neutrophils. J Exp Med $186,739-747$. 
82. Soehnlein, O., et al. (2009) Neutrophil-derived azurocidin alarms the immune system. $J$ Leukoc Biol 85, 344-351.

83. Lemjabbar, H., et al. (1999) Overexpression of alveolar macrophage gelatinase B (MMP-9) in patients with idiopathic pulmonary fibrosis: effects of steroid and immunosuppressive treatment. Am J Respir Cell Mol Biol 20, 903-913.

84. Tester, A.M., et al. (2007) LPS responsiveness and neutrophil chemotaxis in vivo require PMN MMP-8 activity. PLoS One 2, e312.

85. Yamashiro, S., et al. (2001) Phenotypic and functional change of cytokine-activated neutrophils: inflammatory neutrophils are heterogeneous and enhance adaptive immune responses. J Leukoc Biol 69, 698-704.

86. Soehnlein, O., et al. (2010) Phagocyte partnership during the onset and resolution of inflammation. Nat Rev Immunol 10, 427-439.

87. Pelletier, M., et al. (2010) Evidence for a cross-talk between human neutrophils and Th17 cells. Blood 115, 335-343.

88. Stark, M.A., et al. (2005) Phagocytosis of apoptotic neutrophils regulates granulopoiesis via IL-23 and IL-17. Immunity 22, 285-294.

89. Warnatsch, A., et al. (2015) Inflammation. Neutrophil extracellular traps license macrophages for cytokine production in atherosclerosis. Science 349, 316-320.

90. Soehnlein, O., et al. (2015) Neutrophil-macrophage interplay in atherosclerosis: proteasemediated cytokine processing versus NET release. Thromb Haemost 114, 866-867.

91. Sporri, R., et al. (2008) A novel role for neutrophils as critical activators of NK cells. $J$ Immunol 181, 7121-7130.

92. Costantini, C., et al. (2010) Neutrophil activation and survival are modulated by interaction with NK cells. Int Immunol 22, 827-838.

93. Costantini, C., et al. (2011) The defensive alliance between neutrophils and NK cells as a novel arm of innate immunity. J Leukoc Biol 89, 221-233.

94. Zarbock, A., et al. (2007) Platelet-neutrophil-interactions: linking hemostasis and inflammation. Blood Rev 21, 99-111.

95. Hwaiz, R., et al. (2015) Rac1-dependent secretion of platelet-derived CCL5 regulates neutrophil recruitment via activation of alveolar macrophages in septic lung injury. $J$ Leukoc Biol.

96. Hwaiz, R., et al. (2015) Platelet secretion of CXCL4 is Rac1-dependent and regulates neutrophil infiltration and tissue damage in septic lung damage. Br J Pharmacol.

97. Sambrano, G.R., et al. (2000) Cathepsin G activates protease-activated receptor-4 in human platelets. J Biol Chem 275, 6819-6823.

98. Faraday, N., et al. (2013) Cathepsin G-dependent modulation of platelet thrombus formation in vivo by blood neutrophils. PLoS One 8, e71447.

99. Franco, A.T., et al. (2015) Platelets at the interface of thrombosis, inflammation, and cancer. Blood 126, 582-588.

100. Rossaint, J., et al. (2015) Platelets in leucocyte recruitment and function. Cardiovasc Res 107, 386-395.

101. Li, J., et al. (2015) Platelet-neutrophil interactions under thromboinflammatory conditions. Cell Mol Life Sci 72, 2627-2643.

102. Dimasi, D., et al. (2013) Neutrophil interactions with the vascular endothelium. Int Immunopharmacol 17, 1167-1175.

103. Linkermann, A., et al. (2014) Regulated cell death and inflammation: an autoamplification loop causes organ failure. Nat Rev Immunol 14, 759-767.

104. Lande, R., et al. (2011) Neutrophils activate plasmacytoid dendritic cells by releasing self-DNA-peptide complexes in systemic lupus erythematosus. Sci Transl Med 3, 73ra19.

105. Garcia-Romo, G.S., et al. (2011) Netting neutrophils are major inducers of type I IFN production in pediatric systemic lupus erythematosus. Sci Transl Med 3, 73ra20.

106. Kaplan, M.J. (2013) Role of neutrophils in systemic autoimmune diseases. Arthritis Res Ther 15, 219.

107. Kessenbrock, K., et al. (2009) Netting neutrophils in autoimmune small-vessel vasculitis. Nat Med 15, 623-625.

108. Khandpur, R., et al. (2013) NETs Are a Source of Citrullinated Autoantigens and Stimulate Inflammatory Responses in Rheumatoid Arthritis. Sci Transl Med 5, 178 ra140.

109. Serhan, C.N. (2014) Pro-resolving lipid mediators are leads for resolution physiology. Nature 510, 92-101. 
110. Elliott, E.R., et al. (2011) Deletion of Syk in neutrophils prevents immune complex arthritis. J Immunol 187, 4319-4330. 


\section{FIGURE LEGENDS}

\section{Figure 1}

Overview of neutrophil amplification mechanisms

Activated neutrophils can directly enhance their own activity/migration through autocrine and paracrine routes. In addition, they can promote their own functions by modifying the activation state of the complement system, the release of various granule proteases, the formation of neutrophil extracellular traps or influencing the activity of other immune/non-immune cells. Abbreviations: PMN, Polymorphonuclear cell (neutrophil); NETs, Neutrophil extracellular traps.

\section{Figure 2}

Intracellular and autocrine feedback amplification loops of neutrophil function

Neutrophils become polarized upon sensing the chemotactic gradient by forming a leading and a trailing edge. The leading edge is characterized by "frontness" features including a massive $\mathrm{PIP}_{3}$ accumulation through the activation of PI3-kinases (PI3K). This leads to actin polymerization by Rac and activation of further PI3K molecules. Activated Rac also promotes the function of the NADPH oxidase and reactive oxygen species production, resulting in the inhibition of the $\mathrm{PIP}_{3}$-degrading PTEN. Meanwhile, the trailing edge is characterized by "backness" features such as Rho activation and myosin-based contraction. Interestingly, there is a reciprocal inhibition between the "frontness" and "backness" features, amplifying the polarization process. In addition, ATP and its metabolite adenosine further amplifies neutrophil polarization/migration in an autocrine manner. Abbreviations: $\mathrm{PIP}_{3}$, Phosphatidylinositol(3,4,5)-trisphosphate; PI3K, Phosphatidylinositol 3-kinase; PTEN, Phosphatase and tensin homolog; ROCK, Rho-associated protein kinase; ROS, Reactive oxygen species; PMN, Polymorphonuclear cell (neutrophil).

\section{Figure 3}

Paracrine neutrophil amplification circuits

(A) Neutrophils reciprocally interact with other neutrophils by producing the lipid mediator $\mathrm{LTB}_{4}$, through chemokines such as CXCL1, CXCL2 and CCL3, by releasing myeloperoxidase from intracellular granules, or through the release of MRP8/14. (B) Paracrine mechanisms through neutrophil-derived $\mathrm{LTB}_{4}$, chemokines and cytokines lead to feedback amplification of neutrophil recruitment from the vascular space. Release of all those mediators requires Src-family kinases, Syk PLCY2 and Vav-family members, whereas chemokine/cytokine but not $\mathrm{LTB}_{4}$ release is mediated by CARD9. $\mathrm{LTB}_{4}$, Leukotriene $\mathrm{B}_{4}$; MPO, Myeloperoxidase; TLR4, Toll-like receptor 4; Mac-1, Macrophage-1 antigen (CD11b/CD18); PMN, Polymorphonuclear cell (neutrophil).

\section{Figure 4}

Complex feedback amplification loops

(A) Neutrophils activate the complement system by various routes. Activated complement fragments such as $\mathrm{C} 5 \mathrm{a}$ or $\mathrm{C} 3 \mathrm{~b}$ further enhance neutrophil activation/recruitment. (B) Neutrophil granule proteases cleave chemokines and extracellular matrix proteins, resulting in emergence of even more potent 
chemoattractants. (C) Neutrophils promote their own recruitment/activation by the release of different granule molecules and chemokines, which trigger the release of neutrophil-acting chemokines from macrophages and Th17 cells. (D) Neutrophils activate platelets by granule proteins or NET formation, while platelets enhance neutrophil accumulation by direct interaction or chemokine release. (E) Neutrophils bidirectionally interact with fibroblasts and endothelial cells through different cytokines and chemokines. (F) NET formation results in the release of various autoantigens that trigger further autoantibody production and NET release. Abbreviations: PMN, Polymorphonuclear cell (neutrophil); Mac-1, Macrophage-1 antigen (CD11b/CD18); MMP9, Matrix metalloproteinase 9; ECM, Extracellular matrix; PGP, Proline-GlycineProline peptide fragments; MФ, Macrophage; Th17, Th17 cells; NETs, Neutrophil extracellular traps; PSGL-1, P-selectin glycoprotein ligand-1; ANCA, Anti-neutrophil cytoplasmic antibody; ACPA, Anti-citrullinated protein antibody; FcR, Fc receptor. 


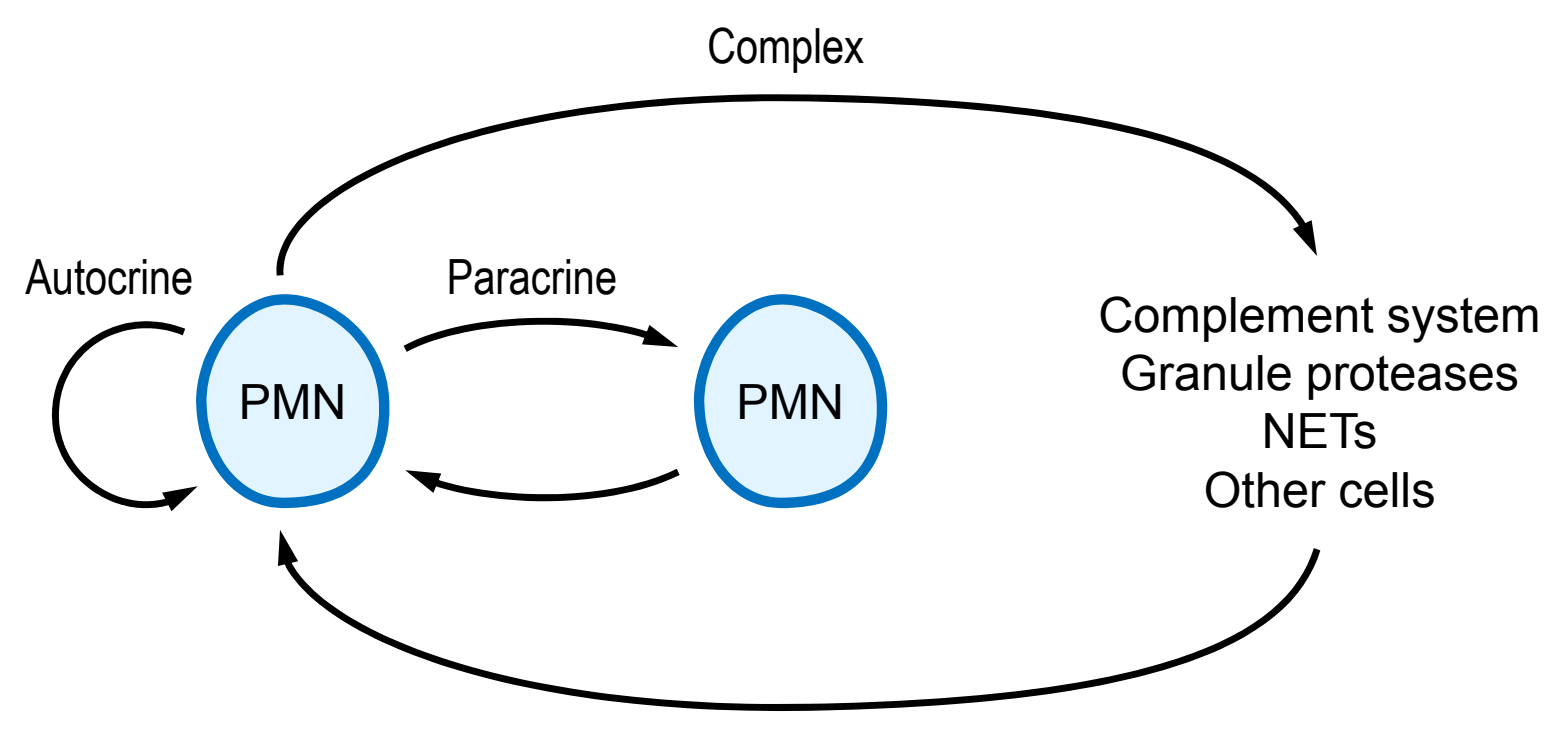




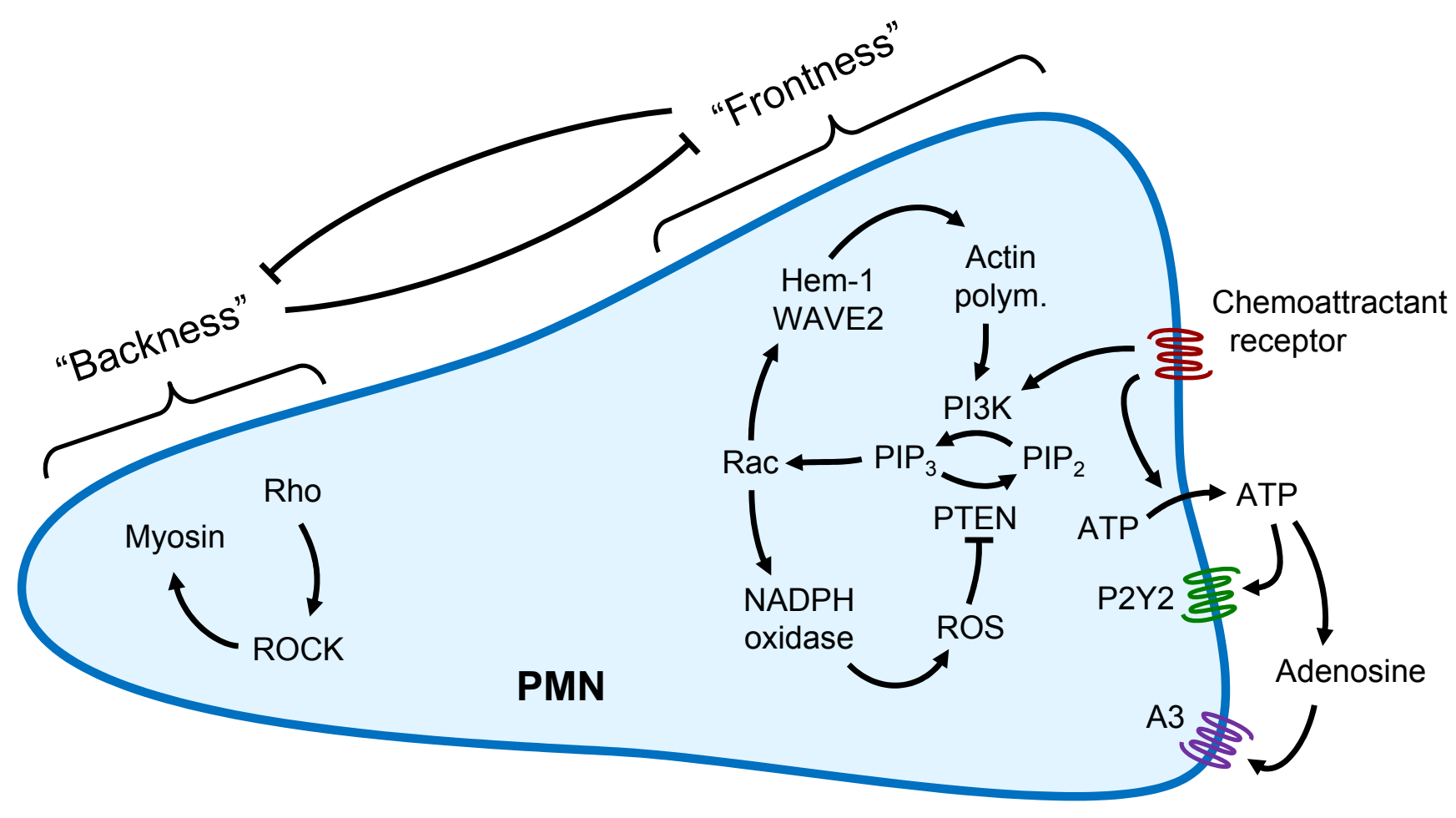



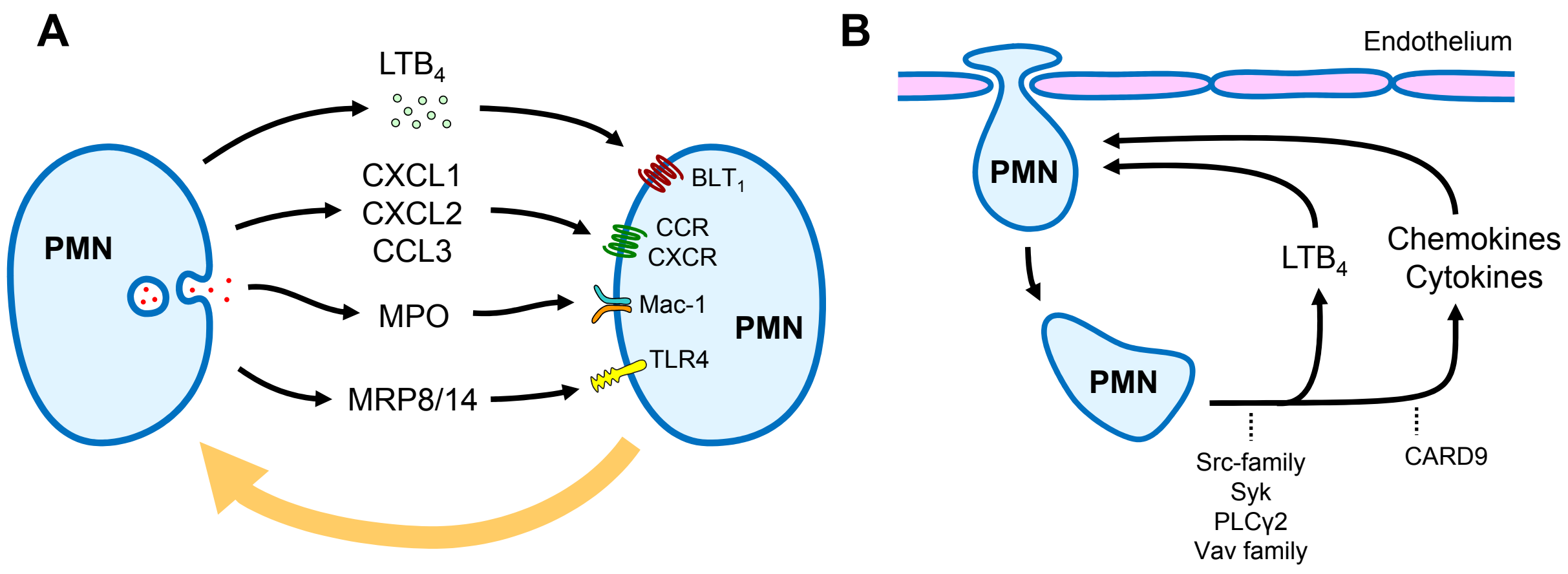
A

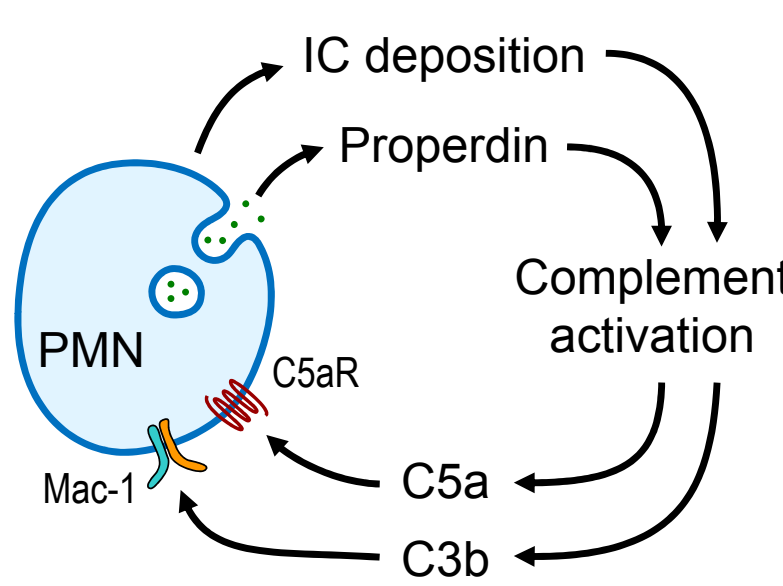

D

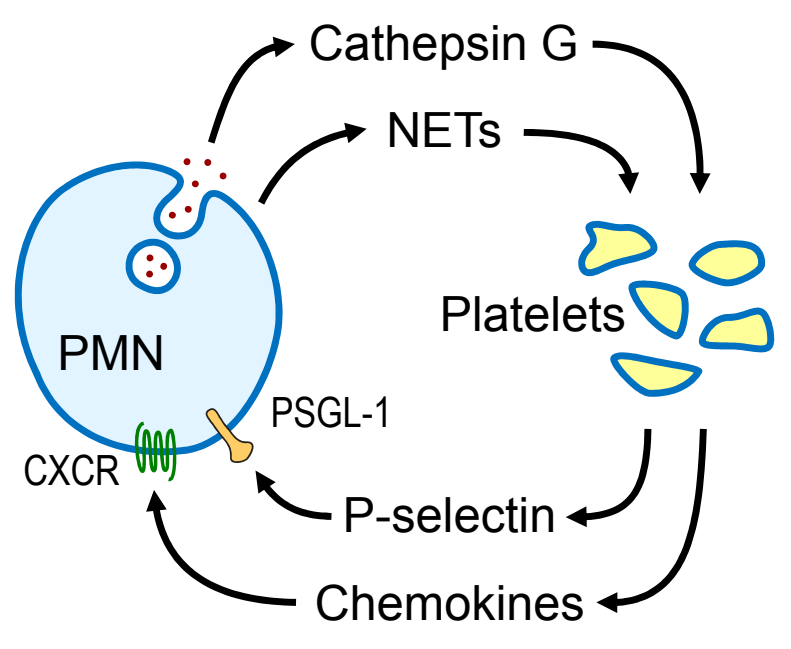

B

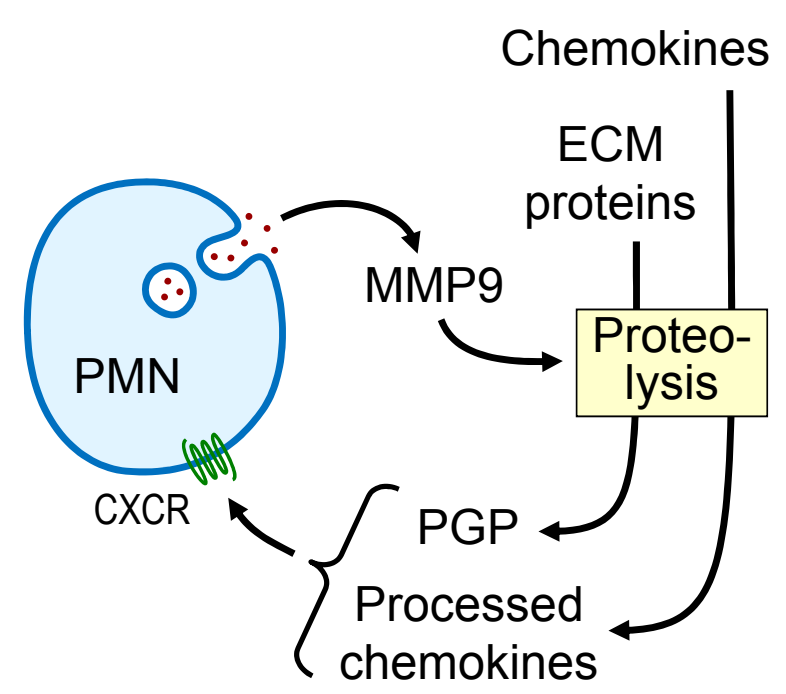

E

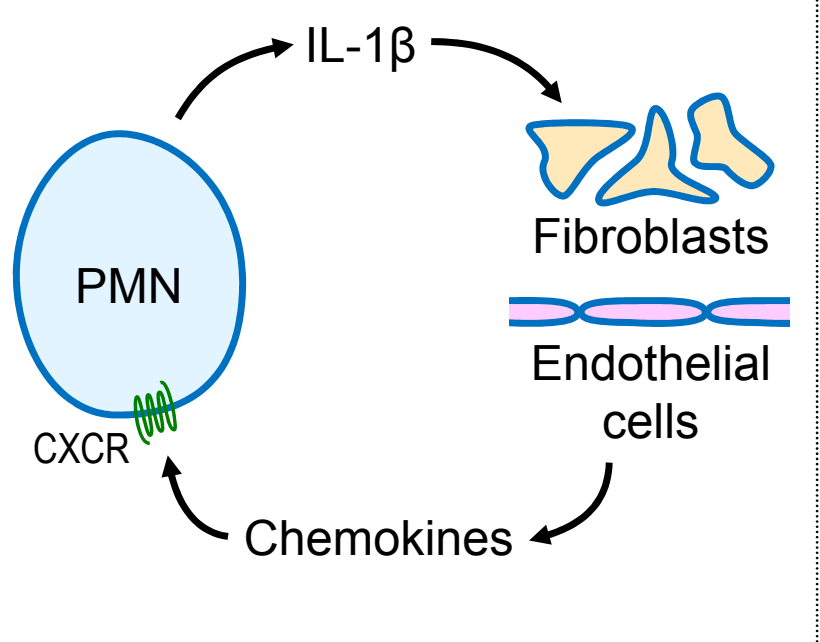

C

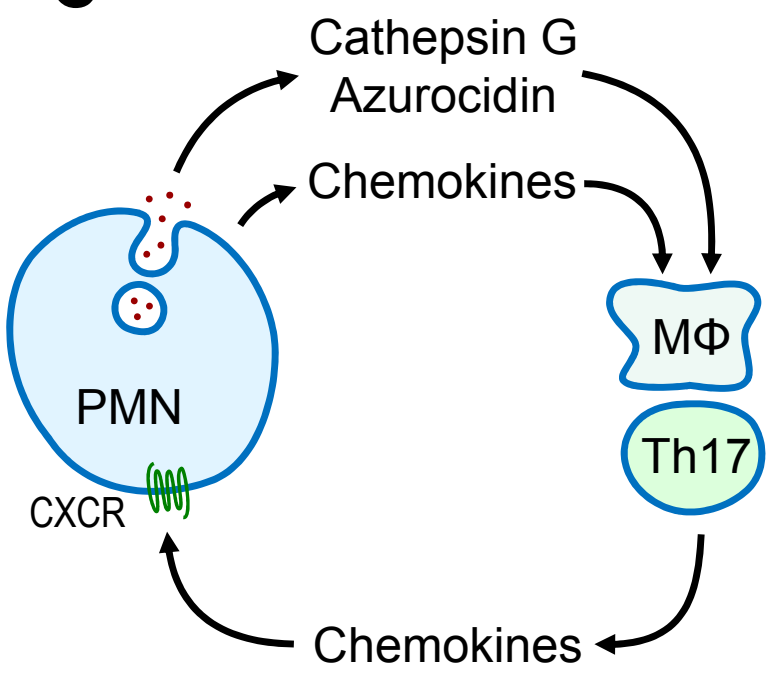

F

NETs

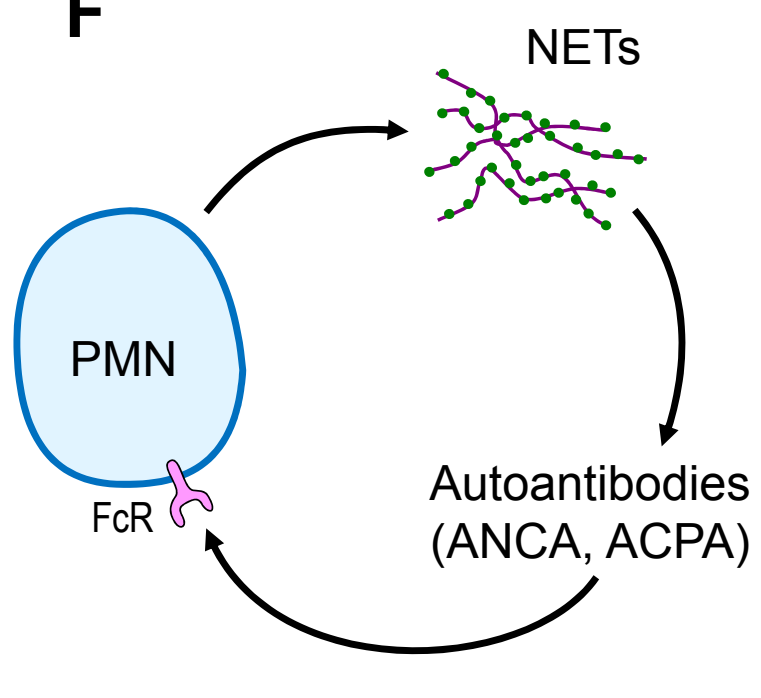

